\title{
Morphology-driven downscaling of Streptomyces lividans to micro-cultivation
}

\author{
Dino van Dissel · Gilles P. van Wezel
}

Received: 4 July 2017/ Accepted: 19 October 2017/Published online: 1 November 2017

(C) The Author(s) 2017. This article is an open access publication

\begin{abstract}
Actinobacteria are prolific producers of secondary metabolites and industrially relevant enzymes. Growth of these mycelial micro-organisms in small culture volumes is challenging due to their complex morphology. Since morphology and production are typically linked, scaling down culture volumes requires better control over morphogenesis. In larger scale platforms, ranging from shake flasks to bioreactors, the hydrodynamics play an important role in shaping the morphology and determining product formation. Here, we report on the effects of agitation on the mycelial morphology of Streptomyces lividans grown in microtitre plates. Our work shows that at the appropriate agitation rates cultures can be scaled down to volumes as small as $100 \mu \mathrm{l}$ while maintaining the same morphology as seen in larger scale platforms. Using image analysis and principal component analysis we compared the morphologies of the cultures; when agitated at 1400-1600 rpm the mycelial morphology in micro-cultures was similar to that obtained in shake flasks, while product formation was also
\end{abstract}

Electronic supplementary material The online version of this article (http://doi.org/10.1007/s10482-017-0967-7) contains supplementary material, which is available to authorized users.

D. van Dissel · G. P. van Wezel ( $₫)$

Molecular Biotechnology, Institute of Biology, Leiden

University, PO Box 9505, 2300RA Leiden, The

Netherlands

e-mail: g.wezel@biology.leidenuniv.nl maintained. Our study shows that the morphology of actinobacteria in micro-cultures can be controlled in a similar manner as in larger scale cultures by carefully controlling the mixing rate. This could facilitate highthroughput screening and upscaling.

Keywords High-throughput screening - Microcultivation $\cdot$ Morphology $\cdot$ Antibiotic $\cdot$ Enzyme $\cdot$ Actinobacteria

\section{Introduction}

Actinobacteria produce a plethora of bioactive natural products, such as antibiotics, anticancer agents, immunosuppressants and antifungals (Barka et al. 2016; Bérdy 2005; Hopwood 2007). In addition, these bacteria produce many industrially relevant enzymes, such as cellulases, amylases and proteases (Vrancken and Anne 2009). Streptomycetes exhibit a complex multicellular life cycle (Claessen et al. 2014). This starts with a single spore that germinates to form vegetative hyphae, which then grow out following a process of hyphal growth and branching to produce a branched vegetative mycelium (Chater and Losick 1997). Nutrient depletion and other environmental stresses induce development, whereby aerial hyphae are formed that differentiate into chains of spores following a complex cell division event whereby ladders of septa are produced within a short time span 
(Jakimowicz and van Wezel 2012; McCormick 2009). The developmental cycle also influences the vegetative mycelia, which partially undergoes programmed cell death to liberate nutrients (Manteca et al. 2005), and chemical differentiation leading to the production of antibiotics and other specialized metabolites (van Wezel and McDowall 2011).

In a submerged environment streptomycetes grow as mycelial networks, typically forming large pellets or clumps. From the industrial perspective, growth as pellets is unattractive, in particular because of masstransfer problems, slow growth and culture heterogeneity (van Dissel et al. 2014; van Wezel et al. 2009). For antibiotic production pellets are generally beneficial, most likely by promoting pathways linked to stress, nutrient limitation and PCD (van Wezel et al. 2006; Martin and Bushell 1996; Manteca et al. 2008).

High throughput (HT) cultivation methods at a small scale are highly desirable, among others to exploit the potential of newly isolated actinobacteria (Kolter and van Wezel 2016). Down-scaling of culture volumes, while maintaining key factors that influence the productivity seen in shake flasks or small scale bioreactors, is necessary to make large screening efforts rapid and economically feasible (Long et al. 2014). However, growing streptomycetes in small cultures is challenging. Streptomycetes typically display a wide range of morphologies in submerged cultures, including dense pellets as well as large mycelial mats [reviewed in van Dissel et al. (2014)]. After inoculation, spores germinate and produce at least two different extracellular polysaccharides (EPS), a cellulose-like polymer catalized by GlxA (Liman et al. 2013; Petrus et al. 2016) and a second EPS synthesized by MatAB (van Dissel et al. 2015). Both polymers induce germling aggregation, and play a key role in pellet formation [Zacchetti et al. (2016) and our unpublished data]. Germling aggregation promotes the formation of pellets, spatially heterogeneous structures with a largely physiologically inactive core, while the peripheral hyphae grow exponentially by tip extension and branching (Celler et al. 2012). The environment also impacts the morphology profoundly. Among others, the composition of the media (Bushell 1988; Glazebrook et al. 1990; Jonsbu et al. 2002), the viscosity (O'Cleirigh et al. 2005) and the pH (Glazebrook et al. 1992) all affect the physiology of the culture, but the hydrodynamics of the culture probably has the most impact
(Olmos et al. 2013). Higher agitation reduces pellet size by promoting fragmentation (Belmar-Beiny and Thomas 1991; Reichl et al. 1992). Fragmentation promotes smaller pellets, thereby increasing the overall growth rate, but increased agitation also results in cell lysis. The relationship between pellet morphology, hydrodynamics (and oxygen supply) and production has been well studied for bioreactors (Tamura et al. 1997; Roubos et al. 2001; Ohta et al. 1995) and for shake flasks (Mehmood et al. 2012; Dobson et al. 2008), but not for smaller cultivation platforms.

To successfully down-scale liquid-grown cultures, the morphology Streptomyces mycelia adopt in larger scale platforms (i.e. shake flasks or bioreactors) should be mimicked as closely as possible. The exact morphology, determined by size, density and shape, also depends on the characteristics of the environment (Wucherpfennig et al. 2010). The hydrodynamics, in other words the characteristics of the agitated medium, is of particular importance as it influences among others the rate of fragmentation (Olmos et al. 2013). Low agitation causes poor distribution of nutrients and reduced oxygen transfer rates, stunting growth and production, while strong agitation can cause cell death (Roubos et al. 2001). Examples of HT cultivation platform for filamentous microorganisms have been described (Minas et al. 2000; Siebenberg et al. 2010; Sohoni et al. 2012). These authors made use of shaken deep-well plates, which results in higher oxygen transfer rates than in small-volume microtitre plates (MTPs) (Duetz et al. 2000). Alternatively the BioLector system with specialized peddle shaped wells allowed the cultivation of 48 parallel 1-ml cultures (Rohe et al. 2012; Huber et al. 2009), which recently was successfully adapted for growth of streptomycetes (Koepff et al. 2017).

In this work we sought to further scale down Streptomyces cultures to $100 \mu \mathrm{l}$ scale. As hosts we used Streptomyces lividans, the preferred enzyme production host (Anné et al. 2012), and the related Streptomyces coelicolor, a model streptomycete for the study of development and antibiotic production (Barka et al. 2016). Cultures were scaled down from shake flasks to $100 \mu$ l cultures, using a digital vortex to obtain the extensive mixing required to control pellet morphology. Using whole slide image analysis, the mycelia were quantified and compared in terms of size and shape. This allowed further optimization of growth in micro-cultures. 


\section{Materials and methods}

Bacterial strains, plasmids

S. lividans 66 (Cruz-Morales et al. 2013) was used for morphological analysis and enzyme production and $S$. coelicolor A3(2) M145 was used for antibiotic production. Plasmid $\mathrm{pIJ703}$, which carries the melC1 and melC2 genes for heterologous tyrosinase production (Katz et al. 1983), was transformed to its host by protoplast transformation (Kieser et al. 2000). Spores were harvested from soy flour mannitol agar plates and stored in $20 \%$ glycerol at $-20{ }^{\circ} \mathrm{C}$ as described (Kieser et al. 2000). The spore titre was determined by plating serial dilutions on SFM agar plates and counting CFUs.

\section{Cultivation conditions}

For cultivation in shake flasks, $S$. lividans was grown in $30 \mathrm{ml}$ tryptic soy broth (Difco) with $10 \%$ sucrose (TSBS) in a $100 \mathrm{ml}$ Erlenmeyer flasks equipped with a stainless steel spring. The flask was inoculated with $10^{6} \mathrm{CFUs} / \mathrm{ml}$ and cultivated at $30{ }^{\circ} \mathrm{C}$ in an orbital shaker with 1 in. orbit (New Brunswick) at 200 RPM. For the production of tyrosinase $25 \mu \mathrm{M} \mathrm{CuCl}_{2}$ was added to the TSBS medium. For antibiotic production S. coelicolor was cultivated in defined glutamate/ glucose based mineral media prepared according to (Wentzel et al. 2012). $100 \mu \mathrm{L}$ media with $10^{6} \mathrm{cfu} / \mathrm{ml}$ spores was added to wells of a V-bottom 96 well MTP (Greiner Bio-One, Germany). To minimize evaporation, the plate was covered with a custom moulded silicone sheet made from MoldMax40 (Materion, USA), using the 96 well plate as a mold and included centred aeration holes with a $2 \mathrm{~mm}$ diameter for each well. An AeraSeal film (Excel Scientific, USA) was added to the top for sterility, while allowing gas exchange. The combined silicone sheet and AeraSeal film were fastened to the plate using masking tape. A Microplate Genie Digital (Scientific Industries, USA) was used for agitation. This microtitre plate vortex has an orbit of $1 \mathrm{~mm}$ with accurate speed control. The rotation speed was confirmed using a Voltcraft DT-10L digital tachometer (Conrad, Germany). The entire setup was placed in a humiditycontrolled incubator set to $70 \% \mathrm{RH}$ and $30^{\circ} \mathrm{C}$. The evaporation rate was around $8 \mu \mathrm{l}$ per well per day. Each condition was performed three times and the shake flask data consist of five independent experiments.

Image and data analysis

Image analysis was performed as described by whole slide imaging combined with automated image analysis using imageJ (Willemse et al. 2017). In short, $100 \mu \mathrm{l}$ sample was transferred to a glass microscope slide and covered by a $24 \times 60$ cover slip. The slide was mounted in an Axio Observer (Zeiss, Germany) equipped with an automated XY-stage, which allowed whole slide imaging using a $10 \times$ objective. The imageJ plugin for automated image analysis optimized for Streptomyces liquid morphology was used to measure particle objects for 12 different features [see ImageJ documentation for mathematical description and (Papagianni 2006) for box surface dimension (BSD) and box mass dimension (BMD)]. Incorrectly analysed pellets (e.g. out-of-focus mycelia) were removed manually. Further data processing was done using Python (Anaconda 4.4.0 distribution). Principal component analysis (PCA) and 2D clustering of the data by a Gaussian mixture model were executed with the included methods in the Scikit-Learn toolbox. 95\% confidence interval (CI) was calculated as: $1.96 \times \operatorname{std} \times n^{-0.5}$. Statistical analysis was performed with the StatsModels Python module using a Tukey's HSD test, with a family-wise error rate set to 0.05 , to assess significant similarities between populations.

Tyrosinase activity measurement

Tyrosinase activity was measured by the conversion over time 1-3,4-dihydroxyphenylalanine spectrophotometrically at a wavelength of $475 \mathrm{~nm}$, as described (van Wezel et al. 2006).

\section{Actinorhodin quantification}

The production of actinorhodin by $S$. coelicolor was determined as follows. Culture supernatant $(40 \mu \mathrm{l})$ was treated with $0.5 \mu \mathrm{l} 5 \mathrm{M} \mathrm{HCl}$ to $\mathrm{pH} 2-3$, extracted with a 0.5 volume of methanol-chloroform (1:1), and centrifuged at $5000 \mathrm{rpm}$ for $10 \mathrm{~min}$. The concentration was calculated from the $A_{542}(\varepsilon 542,18,600)$ (van Wezel et al. 2006). 


\section{Results}

The morphology of S. lividans in shake flasks

To scale down the culture volume, while retaining the morphology, we aimed at replicating key morphological parameters of the liquid-based growth of $S$. lividans in a shake flask, such as pellet formation and fragmentation. We applied the SParticle plugin for ImageJ to quantify the pellets via whole-slide image analysis (Willemse et al. 2017). This plugin allows each particle object to be characterized in 12 different features, allowing multivariate analysis. As a reference, the morphological characteristics of shake flask cultures were investigated. Around 500 aggregates were analysed from five separate $24 \mathrm{~h}$ shake flaskgrown cultures, corresponding to the end of the exponential growth phase, which roughly corresponds to the moment of antibiotic production initiation (Nieselt et al. 2010). The image analysis data obtained from the shake-flask cultures was PCA transformed to find the features that showed the highest variance (Fig. 1a). This revealed that PC 1 was mainly compromised of features that described the size of the pellets and PC 2 consisted of measurements for its circularity (Table S1). Plotting the Feret's diameter versus the circularity indeed shows a nice spread of the data points (Fig. 1b). Previous work comparing the maximum length of pellets revealed two different mycelial populations of $S$. lividans, one forming larger and one smaller pellets (van Veluw et al. 2012). To separate these two populations in both diameter and circularity, the data was clustered using a Gaussian mixture model, creating an accurate description of the morphology. This indeed revealed two distinct clusters of particles that not only differ in size, but also in shape (Fig. 1b). One population (left, green cluster in Fig. 1b) had pellets with similar Feret's diameter of around $87 \mu \mathrm{m}( \pm 10 \%)$, but with a wide standard deviation in circularity (Fig. 1b, green dots and ellipse). Pellets in the second cluster (right, red cluster in Fig. 1b) consisted of larger pellets with an average of $313 \mu \mathrm{m}( \pm 3 \%)$ and were more regularly shaped (Fig. 1b, red dots and ellipse). Because of the effects of pellets on production and regulation it is important to capture all of these morphological characteristics when scaling down.

Influence of agitation rate on pellet morphology

The morphology of liquid-grown cultures of filamentous micro-organisms is a complex process, whereby hydrodynamics play an important role. These determine the shear stress to which the micro-organisms are subjected, affecting mycelial aggregation and fragmentation, besides also regulating the $\mathrm{k}_{\mathrm{L}} \mathrm{a}$, a measure for the rate of gas exchange. The importance of sufficient mixing for filamentous micro-organisms is

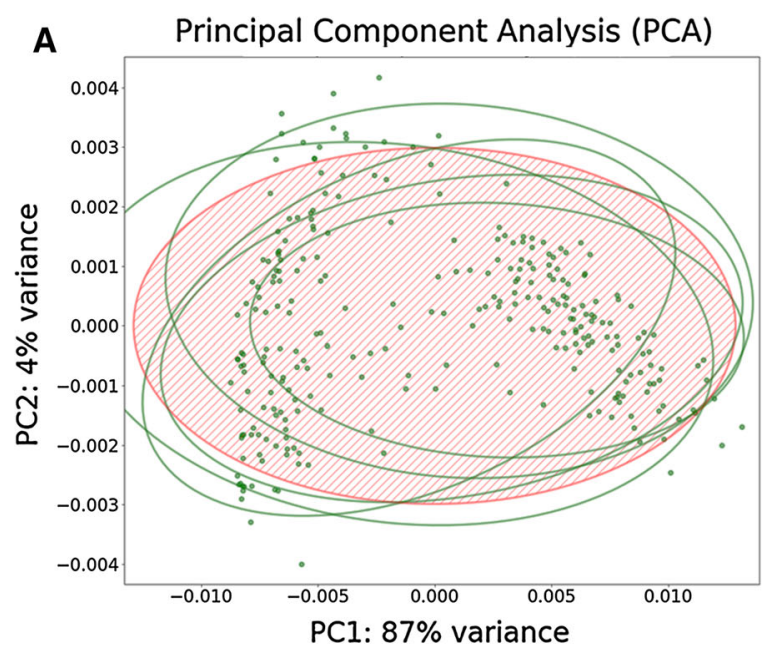

Fig. 1 Multivariate analysis of image analysis data from shake flask cultures. a Principal component analysis; ellipsoids represent $95 \%$ of the data of five independent shake flask

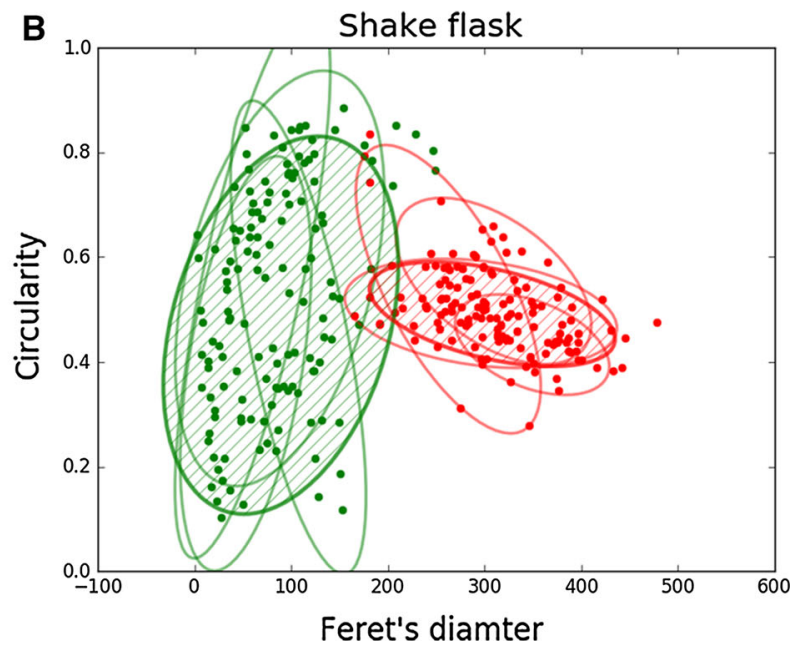

cultures and the red area represents the $95 \%$ of all combined data. b Plot of Feret's diameter and circularity, which shows the two distinct clusters of pellets found in a heterogeneous culture 
exemplified by the need to add a spring or glass beads to a shake flask, which increases the shear stress and promotes growth (Doull and Vining 1989).

We hypothesized that many problems with cultivation of filamentous micro-organisms in small volumes are the result of improper mixing. A digital vortex, designed for microtitre plate (MTP) mixing, allows much higher mixing rates than normally tested and its variable speed settings allow the study of morphology in relation to the agitation rate. This was used to establish whether (and at what agitation rate) a population could be obtained with morphological characteristics similar to those found in larger scale cultures.

Standing cultures of $S$. lividans display a dispersed morphology, which is often entangled with neighbours creating large mats of up to millimeter scale, making them difficult to quantify by automated image analysis (Fig. 2a). When the MTP is agitated at the relative low rate of $600 \mathrm{rpm}$ these large mats are still observed, although hyphal aggregation was already seen (Fig. 2b). This trend continued from 800 to

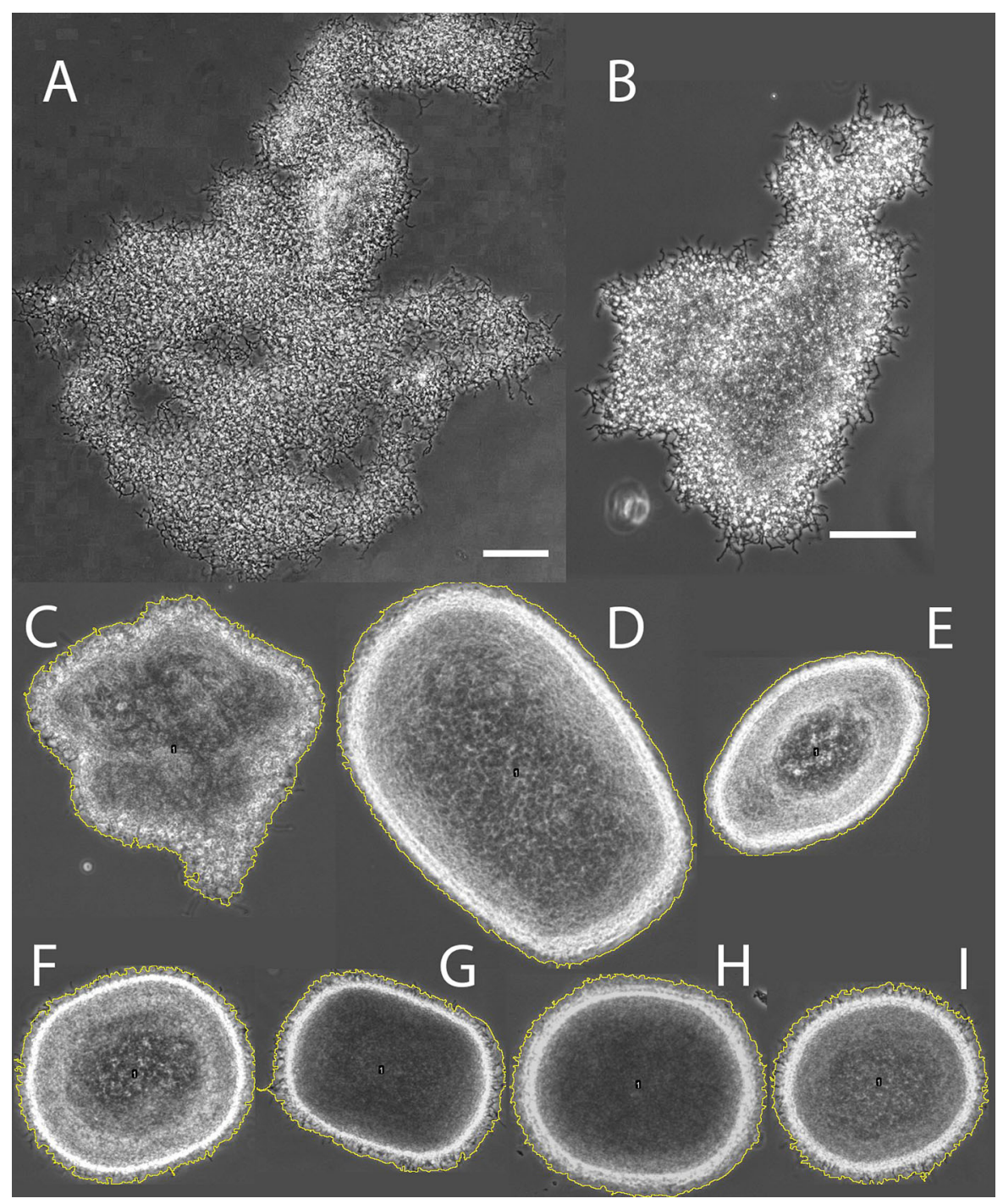

Fig. 2 Representative micrograph of mycelial aggregates found around the centroid of the right cluster, for each cultivation condition. a Standing culture; b-i MTP cultures shaken at b $600 \mathrm{rpm}$, c $800 \mathrm{rpm}$; d $1000 \mathrm{rpm}$; e $1200 \mathrm{rpm}$; f $1400 \mathrm{rpm}$; $1600 \mathrm{rpm}$; h $1800 \mathrm{rpm}$; and i shake flask. Yellow perimeter signifies the measured object by image analysis. Scale bar: $100 \mu \mathrm{m}$ and is shared for B-I. (Color figure online) 
1000 rpm (Fig. 2c, d) where the aggregates became more pellet-like, although still with irregular shapes and large average pellet size as compared to the flaskgrown pellets (Fig. 2i). At $1200 \mathrm{rpm}$ the average pellet size decreased further (Fig. 2e), and at $1400 \mathrm{rpm}$ and higher rates (Fig. 2f, h) we could not visually distinguish the pellets from those obtained from flask-grown cultures (Fig. 2i).
To describe the differences quantitatively, the Feret's diameter and circularity were plotted and clustered to compare them to the shake flask data (Fig. 3; Table 1). This confirmed that pellets obtained from MTP plates shaken at agitation rates of $800 \mathrm{rpm}$ and $1000 \mathrm{rpm}$ share little similarity to those from shake flask cultures, while at higher agitation rates the features are very similar. Where the population of
Fig. 3 Comparison of micro-cultures with shake flasks by clustering of bigaussian population. Feret's diameter and circularity were plotted for 800,1000 , $1200,1400,1600$ and $1800 \mathrm{rpm}$ and clustered into two population with a Gaussian mixture model revealing a population of small (green) and large (red) pellets. Blue ellipsoids represent the average populations found for shake flasks. (Color figure online)
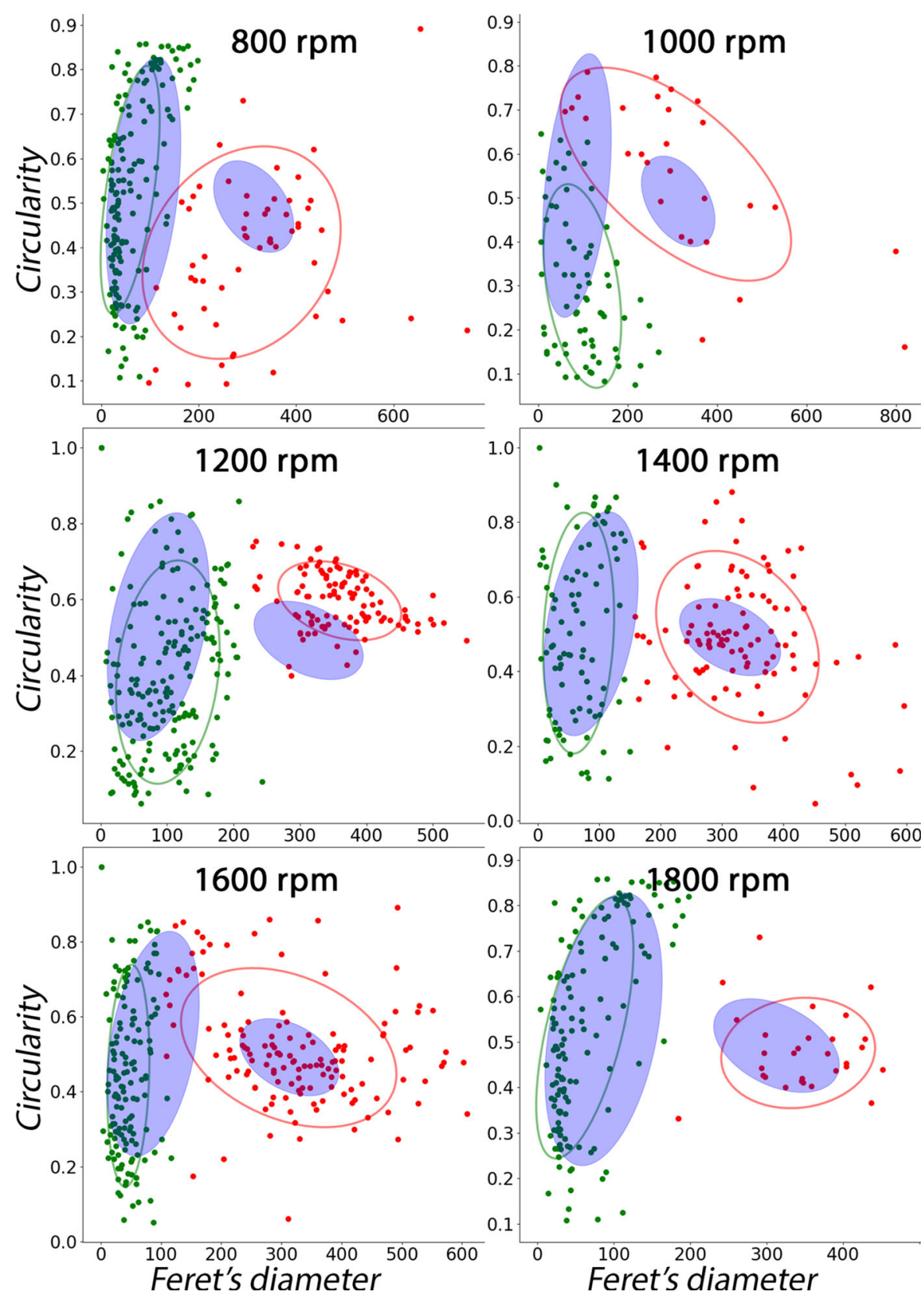
Table 1 Length and circularity of the populations of mycelial aggregates under different growth conditions

\begin{tabular}{lllcccc}
\hline Culture & Feature & $\mu 1(\mu \mathrm{m})$ & $95 \%$ CI $(\mu 1, \mu \mathrm{m})$ & $\mu 2(\mu \mathrm{m})$ & $95 \%$ CI $(\mu 2, \mu \mathrm{m})$ & PF1 $(\%)$ \\
\hline \multirow{2}{*}{ Shake flask } & Feret's diameter & 87 & $78-95$ & 313 & $303-323$ \\
& Circularity & 0.53 & $0.49-0.56$ & 0.49 & $0.48-0.50$ \\
$800 \mathrm{rpm}$ & Feret's diameter & 59 & $54-65$ & 288 & $255-321$ & 75 \\
& Circularity & 0.53 & $0.50-0.56$ & 0.39 & $0.35-0.43$ \\
$1000 \mathrm{rpm}$ & Feret's diameter & 99 & $84-113$ & 312 & $245-378$ \\
& Circularity & 0.30 & $0-0.34$ & 0.55 & $0.49-0.62$ \\
$1200 \mathrm{rpm}$ & Feret's diameter & 101 & $94-108$ & 360 & $348-372$ \\
& Circularity & 0.41 & $0-0.44$ & 0.60 & $0.58-0.61$ \\
$1400 \mathrm{rpm}$ & Feret's diameter & 67 & $59-75$ & 326 & $309-343$ \\
& Circularity & 0.50 & $0.46-0.55$ & 0.49 & $0.46-0.52$ \\
$1600 \mathrm{rpm}$ & Feret's diameter & 46 & $42-50$ & 312 & $293-332$ \\
& Circularity & 0.44 & $0.41-0.48$ & 0.52 & $0.49-0.54$ \\
$1800 \mathrm{rpm}$ & Feret's diameter & 62 & $56-69$ & 341 & $317-366$ \\
& Circularity & 0.53 & $0.50-0.56$ & 0.48 & $0.44-0.51$ \\
\hline
\end{tabular}

CI $95 \%$ confidence level

${ }^{\text {a } P F 1}$ is the participation factor of cluster 1 to the entire population

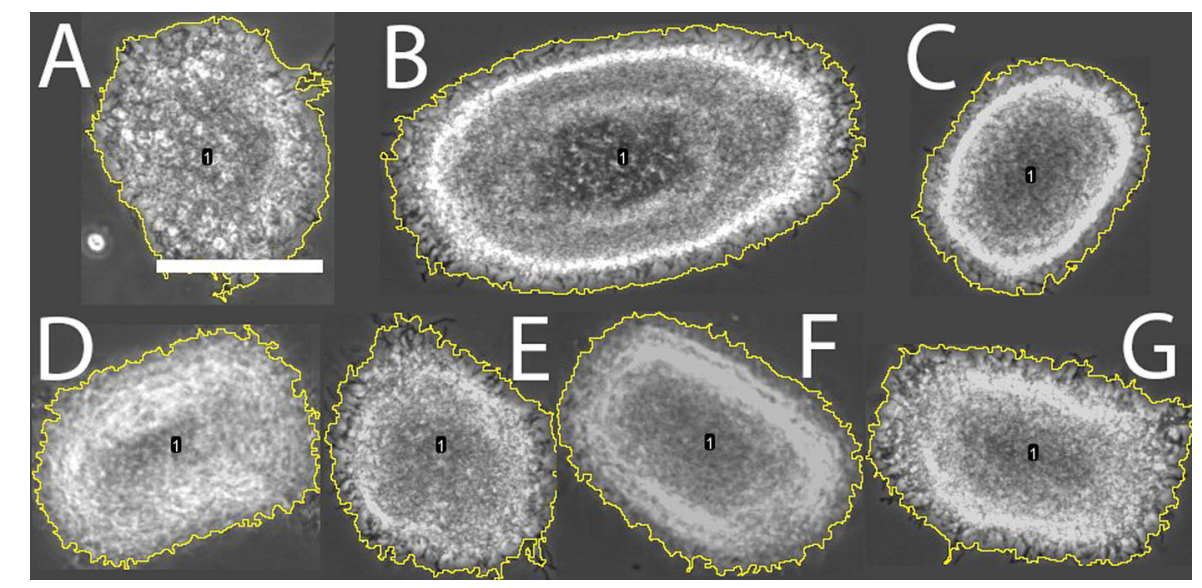

Fig. 4 Representative micrograph of mycelial aggregates found around the centroid of the left cluster, for each cultivation condition. a $800 \mathrm{rpm}$; b $1000 \mathrm{rpm}$; c $1200 \mathrm{rpm}$; d $1400 \mathrm{rpm}$;

small pellets from a shake flask had an average diameter of $85 \mu \mathrm{m}( \pm 10 \%)$ (left and green in Fig. 1 and Fig. 4g), small pellets in MTP cultures grown at $1200 \mathrm{rpm}$ to $1400 \mathrm{rpm}$ had similar averages $(101 \mu \mathrm{m}$ $( \pm 7 \%)$ and $67 \mu \mathrm{m}( \pm 12 \%)$ respectively) (Fig. 4c, d). The sizes of the population of larger pellets also compared well, with an average diameter of $326 \mu \mathrm{m}$ $( \pm 5 \%)$ and $312 \mu \mathrm{m}( \pm 6 \%)$, for 1400 and $1600 \mathrm{rpm}$, respectively, against $313 \mu \mathrm{m}( \pm 3 \%)$ for shake flasks. The average pellet circularity was also very similar e $1600 \mathrm{rpm}$; f $1800 \mathrm{rpm}$ and $\mathbf{g}$ shake flask. Yellow perimeter signifies the measured object by image analysis. Scale bar: $100 \mu \mathrm{m}$. (Color figure online)

between shake flask-grown cultures and MTP-grown pellets at 1400-1800 rpm, although higher variability was seen in MTP-grown pellets (Fig. 3). To statistically assess the similarity, the 12 features measured by image analysis were used to compare each agitation condition with the shake flask data by a Tukey's HSD test (Table 2). Concerning the Feret's diameter and circularity, pellets were similar at 1200-1600, with 1400 and $1600 \mathrm{rpm}$ showing highest similarity when all features are taken into account. 


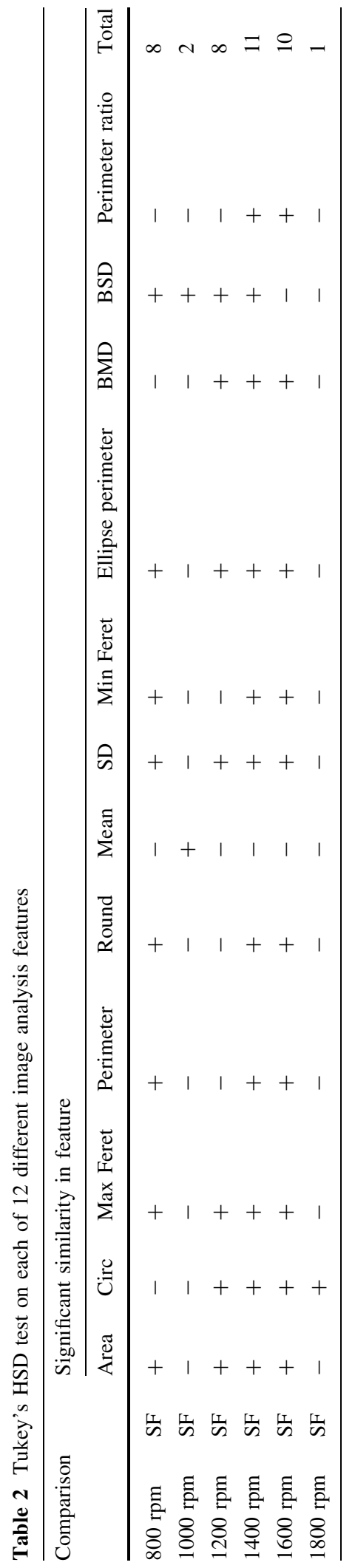

The PCA space calculated for the shake flask data represented the space with the highest variability (Fig. 1a) and hence this was used to analyse MTPgrown cultures for comparison. For this purpose, all data was transformed into the PCA space calculated for the shake flask data (Fig. 5). The transformed data was subjected to a Tukey test for both principal components (Table 3). This again demonstrated that pellets obtained from $100 \mu \mathrm{l}$ MTP cultures agitated at 1400 or $1600 \mathrm{rpm}$ are morphologically very similar to those from shake flask-grown cultures.

Production of heterologous enzymes and antibiotics

The above data indicate that $24 \mathrm{~h}$ old mycelia obtained from MTP cultures and $1400 \mathrm{rpm}$ agitation are similar to those from shake flasks. To obtain insights into how the producing capacity of the two types of cultures compared, we performed pilot experiments on enzyme and antibiotic production. As model system for extracellular enzyme production we used tyrosinase, heterologously expressed in S. lividans by the introduction of plasmid pIJ703 (van Wezel et al. 2006). In line with the similar morphologies, a similar amount of active enzyme was produced in shake flasks (200 rpm, $1 \mathrm{in.}$ orbital) and in MTPs (1400 rpm, $1 \mathrm{~mm}$ orbital), although production started slightly earlier in MTPs (Fig. 6a). As model for antibiotic production S. coelicolor M145 was used. This strain produces the blue-pigmented polyketide antibiotic actinorhodin, which is readily assessed spectrophotometrically. We compared the growth and production of actinorhodin between shake flasks and microcultures grown at $1400 \mathrm{rpm}$. Similar to MTP cultivations with $S$. lividans, $100 \mu$ cultures grown at $1400 \mathrm{rpm}$ were found to be significantly similar by image analysis (Table S2; Fig S1). After $48 \mathrm{~h}$ of growth production of actinorhodin was about $30 \%$ higher in shake flasks than in the microcultures (Fig. 6b). Actinorhodin is a stress-related molecule and its production is that shows strong growth phasedependence. This may explain the slight differences in timing and stress perceived by the mycelia under the different conditions. Taken together, the enzyme and antibiotic assays indicate that production of this antibiotic is comparable between the two culturing methods. 


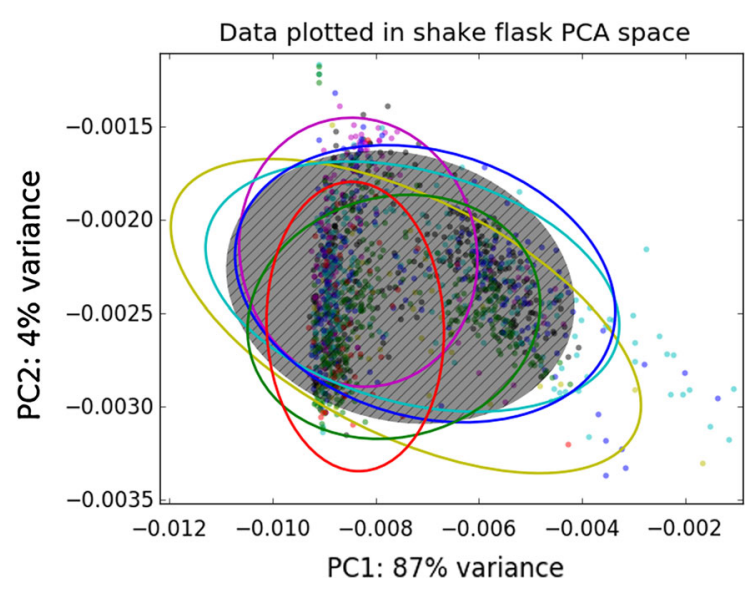

Fig. 5 Comparison of pellets from micro-cultures and shake flask cultivations by PCA. Image analysis data obtained from the microcultures transformed into the PCA space obtained from the shake flask data. Ellipses represent 95\% of the data. Yellow: $800 \mathrm{rpm}$; red: 1000 rpm; green: 1200 rpm; blue: 1400 rpm; cyan: 1600 rpm; magenta: 1800 rpm; black: Shake flask. (Color figure online)

\section{Discussion}

High-throughput screening of actinobacteria for natural products or enzymes typically takes place in micro-scale liquid-grown cultures in an MTP-based setup. The alternative is solid-grown cultures, but it is very difficult to translate growth conditions from solid- to liquid-grown cultures. A drawback of screening of actinobacteria in submerged cultures is the formation of large mycelial networks, which show flocculation or attachment to abiotic surfaces and are associated with slow growth (van Dissel et al. 2014). Additionally, cultures tend to be highly heterogeneous due to the large surface area of the mycelial clumps. Recently, we showed that aggregation of germlings dictates culture heterogeneity (Zacchetti et al. 2016). Because heterogeneity creates a distribution of morphologies, all contributing to production differently (van Veluw et al. 2012; Martin and Bushell 1996), a large population is often required to maintain reproducibility. As heterogeneity is influenced by environmental parameters, careful control is needed to mimic the morphology of a shake flask in small-scale cultivation platform.

Genetically engineered strains have been developed that result in dispersed growth, via over-expression of the cell division activator gene $s s g A$ or deletion of the mat genes that specify the production of an extracellular polysaccharide involved in hyphal aggregation (van Wezel et al. 2006; Traag and Wezel 2008; van Dissel et al. 2015). However, such genetic manipulation may have major consequences for growth and production. Experiments with $S$. coelicolor grown in $1 \mathrm{ml}$ cultures in deep well plates shaken at $300 \mathrm{rpm}$ revealed that while the produced amount was similar as to shake flasks, the growth and actinorhodin production rate were reduced (Minas et al. 2000). However, the morphology was not monitored in this study, so it is unclear if a suboptimal morphology was the reason for the reduced productivity. Growth in 24-square deep well microtitre plates, shaken at $150 \mathrm{rpm}$, required a complex preculture procedure and the addition of glass beads, to obtain reproducible growth, and although growth and production rates were comparable to a bioreactor, the morphology of the organism was completely different, which could cause problems with upscaling later (Sohoni et al. 2012). Recently, the BioLector system was successfully adapted for growth and screening of streptomycetes, which allows parallel growth of 48

Table 3 Tukey's HDS test on MPT data PCA transformed in the shake flask space

\begin{tabular}{|c|c|c|c|c|c|c|c|c|c|}
\hline \multirow[t]{3}{*}{ Comparison } & & \multicolumn{8}{|c|}{ Significant similarity in feature } \\
\hline & & \multicolumn{4}{|l|}{ PC1 } & \multicolumn{4}{|l|}{ PC2 } \\
\hline & & Mean diff & Lower & Upper & Accept & Mean diff & Lower & Upper & Accept \\
\hline $800 \mathrm{rpm}$ & SF & $1.0 \mathrm{E}-03$ & $-7.0 \mathrm{E}-04$ & $1.1 \mathrm{E}-03$ & - & $3.0 \mathrm{E}-04$ & $0.0 \mathrm{E}+00$ & $4.0 \mathrm{E}-04$ & - \\
\hline $1000 \mathrm{rpm}$ & SF & $4.0 \mathrm{E}-04$ & $4.0 \mathrm{E}-04$ & $1.7 \mathrm{E}-03$ & - & $2.0 \mathrm{E}-04$ & $1.0 \mathrm{E}-04$ & $4.0 \mathrm{E}-04$ & - \\
\hline 1200 rpm & SF & $-2.0 \mathrm{E}-04$ & $0.0 \mathrm{E}+00$ & $9.0 \mathrm{E}-04$ & + & $1.0 \mathrm{E}-04$ & $1.0 \mathrm{E}-04$ & $1.0 \mathrm{E}-04$ & - \\
\hline 1400 rpm & SF & $1.0 \mathrm{E}-04$ & $-7.0 \mathrm{E}-04$ & $4.0 \mathrm{E}-04$ & + & $1.0 \mathrm{E}-04$ & $-1.0 \mathrm{E}-04$ & $2.0 \mathrm{E}-04$ & + \\
\hline $1600 \mathrm{rpm}$ & SF & $1.1 \mathrm{E}-03$ & $1.0 \mathrm{E}-04$ & $-4.0 \mathrm{E}-04$ & + & $-1.0 \mathrm{E}-04$ & $0.0 \mathrm{E}+00$ & $2.0 \mathrm{E}-04$ & + \\
\hline 1800 rpm & SF & $2.0 \mathrm{E}-04$ & $1.1 \mathrm{E}-03$ & $6.0 \mathrm{E}-04$ & - & $2.0 \mathrm{E}-04$ & $-2.0 \mathrm{E}-04$ & $0.0 \mathrm{E}+00$ & - \\
\hline
\end{tabular}




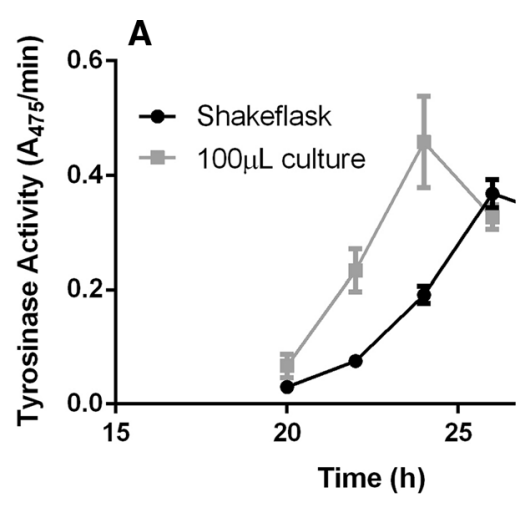

Fig. 6 Comparison of enzyme and antibiotic production. a Tyrosinase production by $S$. lividans 66 . Transformants of $S$. lividans 66 heterologously expressing the secreted enzyme tyrosinase from plasmid pIJ703 were grown in TSBS in either shake flasks or V-bottom MTPs. The graph represents the conversion rate of 1-3,4-dihydroxy-phenylalanine by the culture supernatant, which is indicative of tyrosinase activity. Experiments with shake flasks were done in duplicate, while triplicates were used for MTP-grown cultures. b Actinorhodin

cultures in a MTP with around $1 \mathrm{ml}$ volume (Koepff et al. 2017). This system uses specialized peddleshaped plates for cultivation, and allows the monitoring of $\mathrm{OD}, \mathrm{pH}$ and $\mathrm{DO}$. The authors obtained promising results, obtaining growth parameters that could be compared to those seen in 11 cultures.

In this study, we show that streptomycetes can be successfully cultivated even in $100 \mu \mathrm{l}$ micro-cultures, without the use of specialized equipment or extended pre-culture procedures, while maintaining the same morphology as in large shake flasks. Our data show that the distribution of a heterogeneous mycelial population is highly dependent on the agitation rate in 96-well MTPs. Under the conditions chosen, pellets obtained from $100 \mu \mathrm{l}$ MTP cultures agitated at 1400 or $1600 \mathrm{rpm}$ were morphologically highly comparable to those obtained from shake flask-grown cultures, as shown by image analysis. An initial comparison of the productivity between shake flasks and microcultures revealed comparable yields for the enzyme tyrosinase and the antibiotic actinorhodin produced by S. lividans 66 and S. coelicolor M145 respectively. More extensive studies are required to assess the effect of microcultures on productivity. When required, the level and timing of antibiotic production can be changed by altering the promoter of the pathway-specific regulatory gene that controls the biosynthetic gene cluster of interest (Bibb 2005; van Wezel et al. 2000).

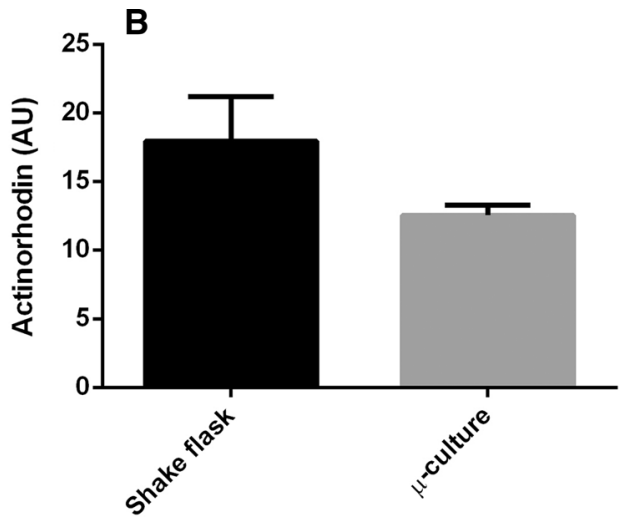

production by $S$. coelicolor M145. S. coelicolor M145 was cultivated in minimal media for $48 \mathrm{~h}$. The shake flasks were run in duplicate, while antibiotic production was measured in three different wells in the MTP. Actinorhodin was extracted by chloroform/methanol and measured spectrophotometrically at $542 \mathrm{~nm}$. The average amount of actinorhodin (in arbitrary units) concentration and the standard error of mean of three independent cultures are shown

At insufficient mixing rates the mycelia failed to aggregate into typical pellet structures. This may at least in part be explained by insufficient oxygen supply. The relationship between oxygen supply and morphology is not well understood, but preliminary experiments where the oxygen supply was limited in a shake flask by reducing the gas exchange, resulted in pellets with a reduced density similar to what was found in poorly agitated MTPs (DvD and GPvW, unpublished results). Although the $\mathrm{k}_{\mathrm{L}} \mathrm{a}$ was not measured in this study, initial calculations using equations for orbital mixing (Seletzky et al. 2007) showed that the oxygen transfer could be lower than adequate, with a $\mathrm{k}_{\mathrm{L}}$ a as low as $40 \mathrm{~h}^{-1}$, for a mixing rate of $800 \mathrm{rpm}$. This low value is suggestive of oxygen limitation as the cause of the morphology observed at low agitation rates and that in part the change in morphology by increased agitation is the result of an increased oxygen supply. While these observations are indicative of oxygen limitation as determining factor for mycelial morphology, oxygen transfer and hydrodynamic stress are coupled processes for orbital shaken cultivation methods (and to some extent also for bioreactors). At least for pristinamycin production hydrodynamic stress, described as the power input, was more descriptive for both pellet morphology and production levels (Mehmood et al. 2012). Thus, downscaling to $100 \mu \mathrm{l}$ is feasible 
for Streptomyces, even if it aggregates into dense pellets. How precisely agitation affects morphogenesis in MTP plates is as yet unclear and requires further study.

Matching the environment, including the physical hydrodynamic forces that determine the morphology is due to its complex nature a difficult task. Our study also illustrates the utility of image analysis to quantify the morphology and assist in the down-scaling process. We devolved a workflow to characterize the morphology of an Streptomyces population, which works in conjunction with the recently published imageJ plugin SParticle, an automated image analysis toolbox to study the morphology of liquid-grown Actinomyces cultures (Willemse et al. 2017). Prior knowledge of the population dynamics of liquid grown cultures, which could be described by a bi-gaussian distribution of Streptomyces particle, was used to characterize shake flask cultures. Comparison of maximal pellet diameter and circularity provides more detailed insights into the exact morphology of the pellets, which aids the down-scaling process. Besides providing the option of medium- to high-throughput screening, the ability to grow Streptomyces with a native morphology on a very small scale also allows studies that involve for example the addition of expensive or low abundance chemicals or enzymes.

\section{Conclusion}

The complex morphology displayed by filamentous actinobacteria in liquid-grown cultures greatly influences their productivity. Screening these bacteria for new therapeutic agents in an MTP-based setup without affecting normal growth and morphology would be a major advantage. This is particularly important in the light of upscaling, so as to maximise the chance that productivity is maintained. We have been able to translate growth and morphology from shake flasks to $100 \mu \mathrm{l}$ micro cultures by carefully tuning the rate of agitation. The resulting growth and average pellet size in standard HTS-compatible MTPs was reproducibly comparable to those in larger scale cultures, which is an important contribution to the state of the art.

Acknowledgements The work was supported by VICI Grant 10379 from the Netherlands Organization for Scientific Research (NWO) to GPvW.
Authors' contributions DD and GPW defined the research theme and designed the experiments. DD carried out the laboratory experiments. DD and GPW analysed the data, interpreted the results and prepared this manuscript. Both authors approved the final manuscript.

\section{Compliance with ethical standards}

Conflict of interest The authors declare that they have no competing interests.

Open Access This article is distributed under the terms of the Creative Commons Attribution 4.0 International License (http:// creativecommons.org/licenses/by/4.0/), which permits unrestricted use, distribution, and reproduction in any medium, provided you give appropriate credit to the original author(s) and the source, provide a link to the Creative Commons license, and indicate if changes were made.

$\begin{array}{ll}\text { Abbreviations } \\ \text { BMD } & \text { Box mass dimension } \\ \text { BSD } & \text { Box surface dimension } \\ \text { CFU } & \text { Colony forming units } \\ \text { CI } & \text { Confidence interval } \\ \text { HTS } & \text { High throughput screening } \\ \mathrm{k}_{\mathrm{L}} \mathrm{a} & \text { Specific oxygen transfer coefficient } \\ \text { MTP } & \text { Microtitre plate } \\ \text { PC } & \text { Principal component } \\ \text { PCA } & \text { Principal component analysis } \\ \text { Rpm } & \text { Rotations per minute } \\ \text { P } & \text { Power dissipation }\end{array}$

\section{References}

Anné J, Maldonado B, Van Impe J, Van Mellaert L, Bernaerts K (2012) Recombinant protein production and streptomycetes. J Biotechnol 158:159-167

Barka EA, Vatsa P, Sanchez L, Gavaut-Vaillant N, Jacquard C, Meier-Kolthoff J, Klenk HP, Clément C, Oudouch Y, van Wezel GP (2016) Taxonomy, physiology, and natural products of the Actinobacteria. Microbiol Mol Biol Rev 80:1-43

Belmar-Beiny MT, Thomas CR (1991) Morphology and clavulanic acid production of Streptomyces clavuligerus: effect of stirrer speed in batch fermentations. Biotechnol Bioeng 37:456-462

Bérdy J (2005) Bioactive microbial metabolites. J Antibiot (Tokyo) 58:1-26

Bibb MJ (2005) Regulation of secondary metabolism in streptomycetes. Curr Opin Microbiol 8:208-215

Bushell ME (1988) Growth, product formation and fermentation technology. Actinomycetes in biotechnology. Academic Press, London, pp 185-217 
Celler K, Picioreanu C, van Loosdrecht MC, van Wezel GP (2012) Structured morphological modeling as a framework for rational strain design of Streptomyces species. Antonie Van Leeuwenhoek 102:409-423

Chater KF, Losick R (1997) Mycelial life style of Streptomyces coelicolor A3(2) and its relatives. In: Shapiro JA, Dworkin $\mathrm{M}$ (eds) Bacteria as multicellular organisms. Oxford University Press, New York, pp 149-182

Claessen D, Rozen DE, Kuipers OP, Sogaard-Andersen L, van Wezel GP (2014) Bacterial solutions to multicellularity: a tale of biofilms, filaments and fruiting bodies. Nat Rev Microbiol 12:115-124

Cruz-Morales P, Vijgenboom E, Iruegas-Bocardo F, Girard G, Yanez-Guerra LA, Ramos-Aboites HE, Pernodet JL, Anne J, van Wezel GP, Barona-Gomez F (2013) The genome sequence of Streptomyces lividans 66 reveals a novel tRNA-dependent peptide biosynthetic system within a metal-related genomic island. Genome Biol Evol 5:1165-1175

Dobson L, O'Cleirigh C, O'Shea D (2008) The influence of morphology on geldanamycin production in submerged fermentations of Streptomyces hygroscopicus var. geldanus. Appl Microbiol Biotechnol 79:859-866

Doull JL, Vining LC (1989) Culture conditions promoting dispersed growth and biphasic production of actinorhodin in shaken cultures of Streptomyces coelicolor A3 (2). FEMS Microbiol Lett 65:265-268

Duetz WA, Ruedi L, Hermann R, O’Connor K, Buchs J, Witholt B (2000) Methods for intense aeration, growth, storage, and replication of bacterial strains in microtiter plates. Appl Environ Microbiol 66:2641-2646

Glazebrook MA, Doull JL, Stuttard C, Vining LC (1990) Sporulation of Streptomyces venezuelae in submerged cultures. J Gen Microbiol 136:581-588

Glazebrook MA, Vining LC, White RL (1992) Growth morphology of Streptomyces akiyoshiensis in submerged culture: influence of $\mathrm{pH}$, inoculum, and nutrients. Can $\mathrm{J}$ Microbiol 38:98-103

Hopwood DA (2007) Streptomyces in nature and medicine: the antibiotic makers. Oxford University Press, New York

Huber R, Ritter D, Hering T, Hillmer AK, Kensy F, Muller C, Wang L, Buchs J (2009) Robo-Lector-a novel platform for automated high-throughput cultivations in microtiter plates with high information content. Microb Cell Fact $8: 42$

Jakimowicz D, van Wezel GP (2012) Cell division and DNA segregation in Streptomyces: how to build a septum in the middle of nowhere? Mol Microbiol 85:393-404

Jonsbu E, McIntyre M, Nielsen J (2002) The influence of carbon sources and morphology on nystatin production by Streptomyces noursei. J Biotechnol 95:133-144

Katz E, Thompson CJ, Hopwood DA (1983) Cloning and expression of the tyrosinase gene from Streptomyces antibioticus in Streptomyces lividans. J Gen Microbiol 129:2703-2714

Kieser T, Bibb MJ, Buttner MJ, Chater KF, Hopwood DA (2000) Practical streptomyces genetics. Int Microbiol 3:260-261

Koepff J, Keller M, Tsolis KC, Busche T, Ruckert C, Hamed MB, Anne J, Kalinowski J, Wiechert W, Economou A, Oldiges M (2017) Fast and reliable strain characterization of Streptomyces lividans through micro-scale cultivation. Biotechnol Bioeng 114:2011-2012

Kolter R, van Wezel GP (2016) Goodbye to brute force in antibiotic discovery? Nat Microbiol 1:15020

Liman R, Facey PD, van Keulen G, Dyson PJ, Del Sol R (2013) A laterally acquired galactose oxidase-like gene is required for aerial development during osmotic stress in Streptomyces coelicolor. PLoS ONE 8:e54112

Long Q, Liu X, Yang Y, Li L, Harvey L, McNeil B, Bai Z (2014) The development and application of high throughput cultivation technology in bioprocess development. J Biotechnol 192:323-338

Manteca Á, Fernández M, Sánchez J (2005) A death round affecting a young compartmentalized mycelium precedes aerial mycelium dismantling in confluent surface cultures of Streptomyces antibioticus. Microbiology 151:3689-3697

Manteca A, Alvarez R, Salazar N, Yagüe P, Sanchez J (2008) Mycelium differentiation and antibiotic production in submerged cultures of Streptomyces coelicolor. Appl Environ Microbiol 74:3877-3886

Martin SM, Bushell ME (1996) Effect of hyphal micromorphology on bioreactor performance of antibiotic-producing Saccharopolyspora erythraea cultures. Microbiol 142:1783-1788

McCormick JR (2009) Cell division is dispensable but not irrelevant in Streptomyces. Curr Opin Biotechnol 12:689-698

Mehmood N, Olmos E, Goergen J-L, Blanchard F, Marchal P, Klöckner W, Büchs J, Delaunay S (2012) Decoupling of oxygen transfer and power dissipation for the study of the production of pristinamycins by Streptomyces pristinaespiralis in shaking flasks. Biochem Eng J 68:25-33

Minas W, Bailey JE, Duetz W (2000) Streptomycetes in microcultures: growth, production of secondary metabolites, and storage and retrieval in the 96-well format. Antonie Van Leeuwenhoek 78:297-305

Nieselt K, Battke F, Herbig A, Bruheim P, Wentzel A, Jakobsen $\varnothing \mathrm{M}$, Sletta H, Alam MT, Merlo ME, Moore J (2010) The dynamic architecture of the metabolic switch in Streptomyces coelicolor. BMC Genomics 11:10

O'Cleirigh C, Casey JT, Walsh PK, O'Shea DG (2005) Morphological engineering of Streptomyces hygroscopicus var. geldanus: regulation of pellet morphology through manipulation of broth viscosity. Appl Environ Microbiol 68:305-310

Ohta N, Park YS, Yahiro K, Okabe M (1995) Comparison of neomycin production from Streptomyces fradiae cultivation using soybean oil as the sole carbon source in an air-lift bioreactor and a stirred-tank reactor. J Ferm Bioeng 79:443-448

Olmos E, Mehmood N, Husein LH, Goergen J-L, Fick M, Delaunay S (2013) Effects of bioreactor hydrodynamics on the physiology of Streptomyces. Bioproc Biosyst Eng $36: 259-272$

Papagianni M (2006) Quantification of the fractal nature of mycelial aggregation in Aspergillus niger submerged cultures. Microb Cell Fact 5:5

Petrus ML, Vijgenboom E, Chaplin AK, Worrall JA, van Wezel GP, Claessen D (2016) The DyP-type peroxidase DtpA is a Tat-substrate required for GlxA maturation and morphogenesis in Streptomyces. Open biology 6:150149 
Reichl U, King R, Gilles ED (1992) Characterization of pellet morphology during submerged growth of Streptomyces tendae by image analysis. Biotechnol Bioeng 39:164-170

Rohe P, Venkanna D, Kleine B, Freudl R, Oldiges M (2012) An automated workflow for enhancing microbial bioprocess optimization on a novel microbioreactor platform. Microb Cell Fact 11:144

Roubos JA, Krabben P, Luiten RGM, Verbruggen HB, Heijnen JJ (2001) A quantitative approach to characterizing cell lysis caused by mechanical agitation of Streptomyces clavuligerus. Biotechnol Progr 17:336-347

Seletzky JM, Noak U, Fricke J, Welk E, Eberhard W, Knocke C, Büchs J (2007) Scale-up from shake flasks to fermenters in batch and continuous mode with Corynebacterium glutamicum on lactic acid based on oxygen transfer and $\mathrm{pH}$. Biotechnol Bioeng 98:800-811

Siebenberg S, Bapat PM, Lantz AE, Gust B, Heide L (2010) Reducing the variability of antibiotic production in Streptomyces by cultivation in 24-square deepwell plates. J Biosci Bioeng 109:230-234

Sohoni SV, Bapat PM, Lantz AE (2012) Robust, small-scale cultivation platform for Streptomyces coelicolor. Microb Cell Fact 11:9

Tamura S, Park Y, Toriyama M, Okabe M (1997) Change of mycelial morphology in tylosin production by batch culture of Streptomyces fradiae under various shear conditions. J Ferment Bioeng 83:523-528

Traag BA, Wezel GP (2008) The SsgA-like proteins in actinomycetes: small proteins up to a big task. Antonie Van Leeuwenhoek 94:85-97

van Dissel D, Claessen D, van Wezel GP (2014) Morphogenesis of Streptomyces in submerged cultures. Adv Appl Microbiol 89:1-45

van Dissel D, Claessen D, Roth M, van Wezel GP (2015) A novel locus for mycelial aggregation forms a gateway to improved Streptomyces cell factories. Microb Cell Fact $14: 44$

van Veluw GJ, Petrus ML, Gubbens J, de Graaf R, de Jong IP, van Wezel GP, Wosten HA, Claessen D (2012) Analysis of two distinct mycelial populations in liquid-grown
Streptomyces cultures using a flow cytometry-based proteomics approach. Appl Microbiol Biotechnol 96:1301-1312

van Wezel GP, McDowall KJ (2011) The regulation of the secondary metabolism of Streptomyces: new links and experimental advances. Nat Prod Rep 28:1311-1333

van Wezel GP, White J, Hoogvliet G, Bibb MJ (2000) Application of redD, the transcriptional activator gene of the undecylprodigiosin biosynthetic pathway, as a reporter for transcriptional activity in Streptomyces coelicolor A3(2) and Streptomyces lividans. J Mol Microbiol Biotechnol 2:551-556

van Wezel GP, Krabben P, Traag BA, Keijser BJF, Kerste R, Vijgenboom E, Heijnen JJ, Kraal B (2006) Unlocking Streptomyces spp. for use as sustainable industrial production platforms by morphological engineering. Appl Environ Microbiol 72:5283-5288

van Wezel GP, McKenzie NL, Nodwell JR (2009) Applying the genetics of secondary metabolism in model actinomycetes to the discovery of new antibiotics. Methods Enzymol 458:117-141

Vrancken K, Anne J (2009) Secretory production of recombinant proteins by Streptomyces. Future microbiology 4:181-188

Wentzel A, Bruheim P, Øverby A, Jakobsen ØM, Sletta H, Omara WAM, Hodgson DA, Ellingsen TE (2012) Optimized submerged batch fermentation strategy for systems scale studies of metabolic switching in Streptomyces coelicolor A3(2). BMC Syst Biol 6:59

Willemse J, Buke F, van Dissel D, Grevink S, Claessen D, van Wezel GP (2017) SParticle, an algorithm for the analysis of filamentous microorganisms in submerged cultures. Antonie Van Leeuwenhoek. https://doi.org/10.1101/159475

Wucherpfennig T, Kiep KA, Driouch H, Wittmann C, Krull R (2010) Morphology and rheology in filamentous cultivations. Adv Appl Microbiol 72:89-136

Zacchetti B, Willemse J, Recter B, van Dissel D, van Wezel GP, Wosten HA, Claessen D (2016) Aggregation of germlings is a major contributing factor towards mycelial heterogeneity of Streptomyces. Sci Rep 6:27045 